\title{
Modesty, Objectification, and Disordered Eating Patterns: A Comparative Study between Veiled and Unveiled Muslim Women Residing in Kuwait
}

\author{
Naif Al-Mutawa ${ }^{a}$ Susannah-Joy Schuilenberg ${ }^{b}$ Rumia Justine ${ }^{b}$ \\ Sarah Kulsoom Taher ${ }^{\mathrm{b}}$ \\ ${ }^{a}$ Faculty of Medicine, Kuwait University, Jabriya, Kuwait; ${ }^{b}$ Soor Center for Professional Therapy and Assessment, \\ Kuwait City, Kuwait
}

\section{Significance of the Study}

- Several studies have been conducted on self-objectification, eating disorders, and their relationship with modesty of clothing with specific reference to the Western population. This study is significant as it has been conducted in a Middle Eastern Muslim population, where modest clothing is mandatory for females. The results of this study could partly remove the misconceptions about modest clothing and other factors responsible for the initiation of eating disorders.

\section{Keywords}

Modesty · Veiling · Objectification · Self-objectification · Eating disorder

\section{Abstract \\ Background: The common thread running through all forms of sexual objectification is the experience of being treated as a body (or collection of body parts) valued predominantly for its use to (or consumption by) others. If girls and women adopt a peculiar view of the self, an effect called self-objec- tification, this may contribute to depression, sexual dysfunc- tion, and eating disorders. The objective of this study is to investigate the impact of modesty (in terms of veiling, i.e., hijab) on objectification, by others and by the self, body im-}

age, and behaviors indicative of eating disturbance in veiled and unveiled Muslim women in Kuwait. Methods: This is a community-based, cross-sectional study conducted through an online survey. The respondents were females living in $\mathrm{Ku}$ wait. Results: Unveiled women experienced more objectification by others than veiled women. There were no significant differences found between veiled and unveiled women in self-objectification and eating disorder symptomatology. There is a significant negative relationship between modesty of clothing and objectification by others. No significant relationship was found between modesty of clothing and the self-objectification and eating disorder scales. For the objectification by others scale, data show that there is a positive relationship between this scale and eating disorders. Eating disorders were found to have a negative relationship

\begin{tabular}{ll}
\hline KARGER & $\begin{array}{l}\text { (e2018 The Author(s) } \\
\text { Published by S. Karger AG, Basel }\end{array}$ \\
E-Mail karger@karger.com & $\begin{array}{l}\text { This is an Open Access article licensed under the Creative Commons } \\
\text { Attribution-NonCommercial-4.0 International License (CC BY-NC) } \\
\text { (http://www.karger.com/Services/OpenAccessLicense), applicable to } \\
\text { the online version of the article only. Usage and distribution for } \\
\text { commercial purposes requires written permission. }\end{array}$
\end{tabular}

Naif Al-Mutawa

Faculty of Medicine

Kuwait University

PO Box 24923, Safat 13110 (Kuwait)

E-Mail naif@al-mutawa.com 
with self-objectification. Conclusion: The results of the study are significant as they clearly demonstrate a weak negative relationship between clothing preferences/affiliations and eating disorders.

C2018 The Author(s)

Published by S. Karger AG, Basel

\section{Introduction}

Religious practice and traditional values in terms of modest dressing are significant prognosticators of eating disorder behaviors. Pedwell [1] uncovered the heterogeneous variations of covering that all fall under the English term "veil." This word refers to a very wide array of women's Islamic dresses around the world, including, but not limited to, "the turban in Turkey, the chador in Iran, the hijab in Britain, the burqa in Afghanistan," and many more international variations of Islamic covering in other regions. The majority of participants cover their hair, waist, stomach, calves, thighs, arms, shoulders, and neck in public; this is in line with social values and practices of modesty in the area. Droogsma [2] suggests that the veil functions to define Muslim identity, perform a behavior check, resist sexual objectification, afford more respect, preserve intimate relationships, and provide freedom. Muslim women who choose to wear the veil argue that an Islamic identity, including the use of traditional clothing and veils, de-emphasizes appearances and protects them from public scrutiny. Moreover, they believe that the Islamic dress protects them from comparisons that they would otherwise be subjected to against unrealistic Western physical ideals [3].

A curvilinear relationship was found between religious orientation and eating attitudes among a subclinical and clinical college population receiving inpatient treatment for eating disorders [4]. On the other hand, Ellison [5] found that the positive influence of religious certainty on wellbeing is direct and substantial. Individuals with strong religious faith experienced higher levels of life satisfaction, greater personal happiness, and fewer negative psychological consequences of traumatic life events. It is often argued that eating disorders are "Western culturebound syndromes" and that individuals from non-Western societies have some immunity against such disorders. Conventional wisdom dictates that it is acculturation, i.e., the influence of, adoption of, and overidentification with Western values and cultures, that has led to the rise of eating disorders in the non-Western world. In the present study, 1,359 of the respondents had lived in a Western country for over 6 months. This serves to elucidate the effect of unrealistic Western ideals that promote thinness and body image comparisons $[1,3,6]$, especially when it comes to the social practice of modesty. A clash between a traditional culture and adopted culture may heighten the risk for eating and body image disturbances in susceptible individuals. The studies, either conducted on Arab populations in Western countries or in Middle Eastern countries, which follow traditional values in terms of modest dressing, have shown either similar or relatively few differences in eating pathology results.

Nobakht and Dezhkam [7] suggested that the prevalence of eating disorders among female adolescents in Tehran is comparable to prevalence rates reported by studies in Western societies. In addition, Gargari et al. [8] found that disordered eating attitudes are just as prevalent among Iranian females. He also found that high social physique anxiety and low self-esteem accompany eating disorders. Ford et al. [9] replicated a study conducted on a Western (i.e., American) population in Egypt; both populations yielded similar results. In fact, the study showed a strong preference for thinness in Arab cultures. Ford et al. [9] recommended that future research be conducted to determine the role of Arab culture in encouraging disordered eating.

Religious affiliation and gender are two additional variables whose importance has been questioned in relation to eating attitudes. In their investigation of Asian and Caucasian adolescents, Ahmad et al. [10] concluded that Muslim adolescents have eating patterns that are congruent with characteristics of eating disorders. The study showed unhealthy eating attitudes and behaviors and high levels of body satisfaction. The eating attitudes of Muslim adolescent males were particularly poor relative to those of adolescent males of other faiths.

The tenets of the objectification theory were tested by examining the role the veil played in the relationship between body image and eating disorder symptoms. Tolaymat and Moradi [11] found, in a sample of 118 Muslim women in the US, that different forms of veiling were negatively related to reports of sexual objectification. In addition, sexual objectification experiences showed a significant positive, indirect, relationship with body surveillance, body shame, and eating disorder symptoms. These relationships are mediated by internalization. Furthermore, internalization of cultural standards of beauty also had a significant positive relationship with eating disorders.

A meditational model of disordered eating, derived from Fredrickson and Roberts' [12] objectification theory, was tested by Noll and Fredrickson [13]. This study
Al-Mutawa et al. 
focused on one aspect of objectification theory, the proposition that self-objectification increases women's experiences of body shame. This in turn may contribute to an increased risk for disordered eating. The study is in agreement with the claims by Fredrickson and Roberts [12]. A culture's practices of female sexual objectification can have profound negative effects on a woman's sense of self, her emotional experiences, and the risk of developing a psychological disorder. Although conducted independently, the study by McKinley and Hyde [14] offers an indirect explanation for the relationship between self-objectification, body shame, and disordered eating that was previously explained by the objectification theory. In studies conducted by Dakanalis et al. [15, 16], it was found that self-objectification was the biggest contributing factor towards eating disorders amongst young women, over other variables such as ideal internalization, body dissatisfaction, dieting, and negative affectivity. Sinclair [17] investigated the relationship between objectification experiences, sociocultural attitudes towards appearance, and objectified body consciousness. Results indicated that there is a significant positive relationship between sociocultural attitudes towards appearance and two of the components of the Objectified Body Consciousness Scale (OBC), i.e., body surveillance and body shame. There was a significant negative relationship between sociocultural attitudes towards appearance and appearance control beliefs. Dakanalis et al. [18] reported the same in another study, which explains how cultural values negatively impact adolescents' perceptions and behaviors concerning their body and the complexities of disordered eating.

This study examines the hypotheses that women who wear the Islamic veil do not experience objectification by others, self-objectification, and eating disturbances. It also tests the hypothesis that there is a relationship between modesty of clothing and 3 other variables, namely objectification by others, self-objectification, and eating disorders.

\section{Materials and Methods}

The study was conducted among 1,573 adult women of various marital status living in Kuwait, fulfilling certain criteria, specifically whether they were Kuwaiti or non-Kuwaiti, Muslim or nonMuslim, and if they had stayed in any Western country for over 6 months. The data collection was conducted online through Survey Monkey, a website used for research. The participants gave their consent after being fully informed about the aims and procedure of the study and that their cooperation was voluntary and confidential. A convenience sample comprising participants from the general population was selected for the study.

Modesty, Objectification, and Disordered Eating Patterns
Objectification Experiences Questionnaire (18 Items)

The Objectification Experiences Questionnaire (OEQ) (18 items) is a self-report instrument designed to measure objectification experiences, such as sexualized inspection and verbal harassment, which are conceptualized as gendered daily hassles that contribute to negative psychological outcomes among women, including dysphoria and depression. It has been taken from the unpublished dissertation of Burnett (1995) [unpubl. data].

The OEQ asks respondents to indicate on a Likert-type scale ranging from 1 , zero occurrences, to 7 , more than 25 occurrences, whether or not they have had certain objectifying experiences, and if so, how frequently these experiences occur in an average month. Because the frequency items were stronger predictors of psychological outcomes in early studies during the development of the OEQ than were the occurrence items, and also because occurrence is essentially subsumed by frequency, Burnett recommended analyzing frequency items.

The OEQ consists of 18 items. Ten objectifying experience items are embedded among 8 other filler questionnaire items that refer to nonobjectifying positive, negative, or neutral experiences. The OEQ correlated more with gender harassment than with more severe forms of sexual harassment, thus providing evidence of convergent validity. Predictive validity was established through moderate to strong correlations between objectification and depression [Burnett, unpubl. data] and between the frequency of objectifying experiences and body image disturbances, disordered eating, and self-objectification $[19,20]$.

Sociocultural Attitudes Towards Appearance Questionnaire

The Sociocultural Attitudes Towards Appearance Questionnaire (SATAQ) was developed to assess a woman's recognition and acceptance of culturally sanctioned standards of thinness and attractiveness [21]. It contains 2 subscales: 6 items assess "Awareness/Acknowledgement of a Societal Emphasis on Appearance," and 8 items assess "Internalization/Acceptance of These Standards." Respondents are asked to rate their agreement/disagreement using a 5-point Likert scale, ranging from "completely disagree" to "completely agree." A higher score on the Awareness subscale reflects greater awareness of the societal emphasis on appearance. A higher score on the Internalization subscale reflects greater personal endorsement or acceptance of societal standards of appearance. A total score is not computed for the SATAQ; rather a score for Awareness is computed, along with a score for Internalization.

\section{Sociocultural Attitudes Towards Appearance Scale-3}

(30 Items)

The Sociocultural Attitudes Towards Appearance Scale-3 (SATAQ-3) (30 items) subscales have been shown to have excellent convergent validity with measures of body image and eating disturbance. The SATAQ-3 measures multiple aspects of a societal influence and should prove useful for basic risk factor work as well as for gauging the efficacy of prevention and treatment programs. It also measures media influence related to sports, athleticism, or exercise.

\section{Objectified Body Consciousness Scale (24 Items)}

The OBC (24 items) is a self-report measure that asks participants to respond to statements using a 6-point Likert-type scale ranging from "strongly agree" to "strongly disagree." The OBC is 
Table 1. Demographic characteristics

\begin{tabular}{lc}
\hline Nationality & \\
Kuwaiti & $1,116(70.9)$ \\
Non-Kuwaiti & $416(26.4)$ \\
Missing & $41(2.6)$ \\
Religion & \\
Muslim & $1,359(86.4)$ \\
Non-Muslim & $165(10.5)$ \\
Missing & $49(3.1)$ \\
Staying in any Western country for more than 6 months \\
Yes & $1,359(76.7)$ \\
No & $165(19.3)$ \\
Missing & $49(4.1)$ \\
Mean age \pm SD, years & $28.09 \pm 7.95$ \\
\hline
\end{tabular}

Values are $n(\%)$ unless otherwise indicated. SD, standard deviation.

Table 2. Comparison of veiled and unveiled women with respect to the objectification by others scale

\begin{tabular}{lllrl}
\hline & \multirow{2}{*}{$n$} & \multicolumn{3}{c}{ Objectification by others scale } \\
\cline { 3 - 5 } & & mean & \multicolumn{1}{c}{ SD } & \multicolumn{1}{c}{$p$} \\
\hline Veiled women & 661 & 31.24 & 9.78 & $-6.61^{* * *}$ \\
Unveiled women & 382 & 35.58 & 10.50 & \\
\hline
\end{tabular}

SD, standard deviation. ${ }^{* * *} p<0.001$.

composed of 3 separate but related 8-item subscales: (a) Surveillance (i.e., viewing the body as an outside observer); (b) Body Shame (i.e., feeling shame when the body does not conform to accepted societal standards), and (c) Appearance Control Beliefs (i.e., the amount of perceived control a woman believes she has over her appearance).

High scores on Surveillance indicate frequent surveillance of appearance and thoughts of the body in terms of how it looks, whereas a low score indicates rare surveillance and thoughts of the body in terms of how it feels [14]. High scores on Body Shame reflect feelings about the body that are consistent with cultural norms (e.g., feeling unattractive if overweight) and increased shame. High scores on Appearance Control Beliefs indicate the belief that weight and appearance can be controlled if one tries hard enough.

Eating Disorder Inventory Body Dissatisfaction and Drive for Thinness Subscales

The Eating Disorder Inventory [22] Body Dissatisfaction (EDI-BD) is a 7-item subscale that assesses overall satisfaction with various weight-related body sites. It has demonstrated good reliability across varied samples in previous studies [22]. The Eating Disorder Inventory Drive for Thinness (EDI-DT) assesses restriction of intake, the desire to be thin, and the fear of gaining weight. It has demonstrated adequate reliability in non-patient and eating- disordered samples [22]. This scale has been validated on patients with eating disorders as well as the general population for treatment planning and evaluation of eating-related problems [23].

Modesty of Clothing 5-Point Likert Scales

The Modesty of Clothing 5-point Likert scales were used to assess how frequently participants reported wearing clothing to cover particular body parts when in public. Thirteen body parts were assessed, including hair, neck, shoulders, upper arms, lower arms, waist, thighs, legs, and feet.

\section{Statistical Analysis}

Statistical analyses were conducted using PASW (SPSS version 18). Comparisons between the groups of women with hijab and women without hijab were made using an independent $t$ test. The matrix correlation was used to find correlations between the variables modest dressing, sexual objectification, objectification from others, and eating disorders.

\section{Results}

\section{Sample Description}

$70.9 \%$ of the respondents were Kuwaitis and $26.4 \%$ were non-Kuwaitis. $86.4 \%$ were Muslims and $10.5 \%$ were non-Muslims. $76.7 \%$ of the respondents had stayed in Western countries for more than 6 months, while $19.3 \%$ had not. The age average of the sample was 28.09 (SD 7.95) years (Table 1$)$.

\section{Testing of Hypotheses}

Women Who Wear the Islamic Veil Do Not

Experience Objectification by Others

There is a significant difference between veiled and unveiled women in the objectification by others scale. Unveiled women have a higher mean score (mean 35.58, SD 10.50) than unveiled women (mean 31.24, SD 9.78) on objectification by others $(p<0.001)$ (Table 2$)$.

Women Who Wear the Islamic Veil Do Not

Experience Self-Objectification

There is no significant difference in the experience of self-objectification between veiled and unveiled women (mean 43.34, SD 14.68 and mean 44.44, SD 14.36, respectively; $p<-1.21)$ (Table 3$)$.

\section{Women Who Wear the Islamic Veil Do Not}

Experience Eating Disturbances

Table 4 shows that no significant differences were found between veiled and unveiled women on the eating disorder symptomatology scale and all other 4 subscales: eating concern, shape concern, weight concern, and dietary restraint.
Al-Mutawa et al. 
There Are Relationships between Modesty

of Clothing and Objectification by Others,

Self-Objectification, and Eating Disorders

There is a significant negative relationship between modesty of clothing and objectification by others $(r=$ $-0.210 ; p<0.01$ ). The higher the mean on the modesty of clothing scale, the less the participants experience objectification by others. No significant relationships were found between modesty of clothing and the self-objectification and eating disorders scales. For the objectification by others scale, data show that there is a positive relationship between this scale and eating disorders $(r=$ $0.196 ; p<0.01)$. The higher the mean on the objectification by others scale, the higher the risk of eating disorders. Eating disorders are found to have a negative relationship with self-objectification $(r=-0.121 ; p<0.01)$. The higher the mean on the self-objectification scale, the lower the incidence of eating disorders (Table 5).

\section{Discussion}

The present study aimed to further the literature in the area of modesty and its implications for perceived objectification of women and eating behaviors. The first aim of the study was to find out whether women who wear the Islamic veil experience objectification by others. The results indicate that veiled women experience less objectification by others than unveiled women. Hence, modesty of clothing was found to have a significant impact on the objectification experiences of women, whereas the less covered a woman is, the more she is objectified by others.

The traditional notion that the veil symbolizes the oppression of women, in terms of freedom and identity, has been argued against by many others, stating that wearing the veil de-emphasizes appearance and acts as a protection against public scrutiny. In addition, it shields women from unrealistic Western ideals, which promote thinness and body image comparisons $[6,1,3]$. The outcome of the present study supports the fact that by being veiled a woman is less likely to encounter situations that would constitute objectification by others, such as gazing, leering, comments about a woman's body, evaluating a woman's physical appearance, whistling, or honking at female passersby.

The second aim of the study was to find out whether modesty has an effect on women's own perceptions of their objectification. The results suggest that there is no significant difference between the 2 groups (veiled and unveiled women) in terms of self-objectification experiences. This implies that wearing the Islamic veil does not
Table 3. Comparison of veiled and unveiled women with respect to the self-objectification scale

\begin{tabular}{lllll}
\hline & $n$ & \multicolumn{3}{c}{ Self-objectification scale } \\
\cline { 3 - 5 } & & mean & SD & $p$ \\
\hline Veiled women & 709 & 43.34 & 14.68 & -1.21 \\
Unveiled women & 403 & 44.44 & 14.36 & \\
\hline
\end{tabular}

SD, standard deviation.

Table 4. Comparison of veiled and unveiled women with respect to the eating disorder symptomatology scales and 4 subscales (eating concern, shape concern, weight concern, and dietary restraint)

\begin{tabular}{lcccc}
\hline & $n$ & \multicolumn{3}{l}{$\begin{array}{l}\text { Eating disorder } \\
\text { symptomatology }\end{array}$} \\
\cline { 3 - 5 } & & mean & SD & $p$ \\
\hline Veiled women & 427 & 67.89 & 34.54 & -0.499 \\
Unveiled women & 255 & 69.30 & 37.62 & \\
\hline Eating concern & & & & \\
$\quad$ Veiled women & 510 & 39.68 & 18.04 & -1.33 \\
$\quad$ Unveiled women & 289 & 41.46 & 18.54 & \\
\hline $\begin{array}{l}\text { Shape concern } \\
\text { Veiled women }\end{array}$ & 503 & 19.59 & 0.910 & 0.040 \\
Unveiled women & 291 & 20.41 & 1.24 & \\
\hline Weight concern & & & & \\
$\quad$ Veiled women & 460 & 5.32 & 3.10 & 0.109 \\
$\quad$ Unveiled women & 266 & 5.29 & 3.18 & \\
\hline Dietary restraint & & & & \\
$\quad$ Veiled women & 495 & 19.30 & 12.14 & -1.76 \\
$\quad$ Unveiled women & 280 & 20.85 & 11.34 & \\
\hline
\end{tabular}

$\mathrm{SD}$, standard deviation.

Table 5. Matrix correlation on modesty of clothing and objectification by others, self-objectification, and eating disorders

\begin{tabular}{llllll}
\hline & 1 & 2 & 3 & 4 \\
\hline 1 & Modesty of clothing & - & $-0.210^{* *}$ & -0.010 & -0.009 \\
2 & Objectification by others & - & - & -0.021 & $0.196^{* *}$ \\
3 & Self-objectification & - & - & - & $-0.121^{* *}$ \\
4 & Eating disorders & - & - & - & - \\
\hline \multirow{2}{*}{$* * 0.01}$. & & & & \\
\hline
\end{tabular}


have an impact on the self-image perceptions of women. These findings are in line with previous results which indicated that no significant body image differences existed between Muslim and non-Muslim women [24]. However, the path analyses of a previous study comparing Muslim and non-Muslim women have suggested negative relationships between strength of faith and body dissatisfaction and self-objectification [24]. This finding could be explained by the fact that the study compared Muslims with non-Muslims, whereas the present study looked at Muslim women only. Therefore, the lack of significant results in the present study could be attributable to factors within the Muslim faith. Religion accredits self-worth independently of body image and appearance and instead places emphasis on moral and religious pursuits [25].

The third aim of the study was to explore whether wearing the Islamic veil has an effect on the prevalence of eating disorders in women. We found that there were no significant differences between the 2 groups on any of the scales of eating disorder symptomatology (eating concern, shape concern, weight concern, and dietary restraint). Although previous studies have proposed that Islamic traditions, such as fasting during the month of Ramadan, could normalize the views on restricted eating and contribute to unhealthy diets and weight loss regimes in women, the present evidence suggests the contrary [3].

The final aim of the study was to look for other relationships between the variables being studied (i.e., modesty of clothing, self-objectification, objectification by others, and eating disorders). The results indicated that modesty of clothing had a significant negative relationship with objectification by others. It is noteworthy that our findings show that being veiled accounts for a substantial difference in the experience of objectification by others. These results are similar to the findings from the first hypothesis, where women who were more covered experienced less objectification from others than those who were less covered. In turn, objectification by others has shown a significant positive relationship with eating disorders. The results of previous studies on modesty effects confirm the probability of eating disorders decreasing with modesty. Fredrickson et al. [26] experimentally created an environment where self-objectification was induced by having participants try on either a sweater or a swimsuit. They found that wearing the swimsuit elicited increased body shame, which in turn predicted restrained eating behavior. In addition, Tolaymat and Moradi [11] found that experience of objectification by others had a significant positive indirect relationship with body surveillance, body shame, and eating disorders, through the mediating role of internalization. The internalization of cultural standards of beauty also had a significant positive relationship with eating disorders. Susov [27] determined that extrinsic, rather than intrinsic, religious orientation is predictive of bulimic symptomology. In line with the results of the present study, participants who experience high rates of objectification by others also exhibit high binge eating symptomology. Researchers argue that there are a number of pressures that are now placed on women on a global level, which include contradicting cultural and religious expectations. These social situations tend to have an impact on women's sense of their body [28].

The present study also found that eating disorders had a significant negative relationship with self-objectification. This goes against the traditional views and previous literature $[15,16,18]$, which expect women who objectify themselves to have symptoms of eating disorders. This result could be explained by the fact that the majority of the respondents were from a Muslim orientation. An intrinsic focus on religious values and the derivation of one's sense of self from them could result in healthy attitudes towards one's body image, and hence eating habits [27]. Fredrickson and Roberts suggest that "disordered eating is largely contingent on women adopting an observer's perspective of the body, not simply whether women perceive that they are being objectified" [12]. This feeds into the theory that perhaps self-objectification is a more effective predictor of eating disturbance than objectification by others. However, when exposed to objectification by others, not all women react similarly and are engaged in self-objectification [29].

It is important to note that there appears to be a relationship between objectification by others and eating disorders mediated by self-objectification. The present study could direct professionals in the field on the right course to establish interventions for their female clients suffering from eating disorders. While modesty was not directly correlated with eating disorders in women, it was related to objectification by others, which is significantly correlated with eating disorders. This sheds light on the sociocultural influence on eating disorders. Therapists and other health-care professionals can use this information to start discussions with their future patients regarding perceptions of their clothing preferences and how they affect their disordered eating behaviors.

The study was conducted in a large sample, which is a strength. Regarding the limitations of the study, the study did not take differences between Kuwaiti and other women into consideration and had a combined sample of veiled and unveiled women of various nationalities rather than a single nationality.
Al-Mutawa et al. 


\section{Conclusion}

No significant relationships were found between modesty of clothing and the self-objectification and eating disorders scales. Further research into religion, impact of acculturation, modesty, strength of faith, and the veil would provide a deeper analysis of what connects these variables in order to understand how to make preventions and to make treatment efforts for individuals experiencing eating disturbances in nonclinical and clinical populations more successful.

\section{Statement of Ethics}

All procedures followed were in accordance with the ethical standards of the responsible committee on human experimentation (institutional and national) and with the Helsinki Declaration of 1975, as revised in 2000. Informed consent to be included in this study was obtained from all patients.

\section{Disclosure Statement}

There are no conflicts of interest to declare.

\section{References}

1 Pedwell C. Tracing "the anorexic" and "the veiled woman": towards a relational approach. LSE Gender Institute Working Paper Series. 2007;20.

2 Droogsma RA. Redefining hijab: American Muslim women's standpoints on veiling. J Appl Commun Res. 2007;35(3):294-319.

3 Ahmed L. Women and gender in Islam: historical roots of a modern debate. Yale University Press; 1992.

4 Smith MH, Richards PS, Maglio CJ. Examining the relationship between religious orientation and eating disturbances. Eat Behav. 2004 May;5(2):171-80.

5 Ellison CG. Religious involvement and subjective well-being. J Health Soc Behav. 1991 Mar;32(1):80-99.

6 Hirschmann NJ. Western feminism, eastern veiling and the question of free agency. Constellations. 1998;5(3):345-68.

7 Nobakht M, Dezhkam M. An epidemiological study of eating disorders in Iran. Int J Eat Disord. 2000 Nov;28(3):265-71.

8 Gargari BP, Khadem-Haghighian M, Taklifi E, Hamed-Behzad M, Shahraki M. Eating attitudes, self-esteem and social physique anxiety among Iranian females who participate in fitness programs. J Sports Med Phys Fitness. 2010 Mar;50(1):79-84.

9 Ford KA, Dolan BM, Evans C. Cultural factors in the eating disorders: a study of body shape preferences of Arab students. J Psychosom Res. 1990;34(5):501-7.

10 Ahmad S, Waller G, Verduyn C. Eating attitudes and body satisfaction among Asian and Caucasian adolescents. J Adolesc. 1994;17(5): 461-70.

11 Tolaymat LD, Moradi B. U.S. Muslim women and body image: links among objectification theory constructs and the hijab. J Couns Psychol. 2011 Jul;58(3):383-92.
12 Fredrickson BL, Roberts TA. Objectification theory: toward understanding women's lived experiences and mental health risks. Psychol Women Q. 1997;21(2):173-206.

13 Noll SM, Fredrickson BL. A mediational model linking self-objectification, body shame and eating disorder. Psychol Women Q. 1998;22(4):623-36.

14 McKinley NM, Hyde J. The objectified body consciousness scale. Psychol Women Q. 1996; 20(2):181-215.

15 Dakanalis A, Clerici M, Bartoli F, Caslini M, Crocamo C, Riva G, et al. Risk and maintenance factors for young women's DSM-5 eating disorders. Arch Women Ment Health. 2017 Dec;20(6):721-31.

16 Dakanalis A, Timko A, Serino S, Riva G, Clerici M, Carrà G. Prospective psychosocial predictors of onset and cessation of eating pathology amongst college women. Eur Eat Disord Rev. 2016 May;24(3):251-6.

17 Sinclair SL. Object lessons: A theoretical and empirical study of objectified body consciousness in women. J Ment Health Couns. 2006;28(1):48-68.

18 Dakanalis A, Carrà G, Calogero R, Fida R, Clerici M, Zanetti MA, et al. The developmental effects of media-ideal internalization and self-objectification processes on adolescents' negative body-feelings, dietary restraint, and binge eating. Eur Child Adolesc Psychiatry. 2015 Aug;24(8):997-1010.

19 Brownlow BS. The relationship between objectification, body image disturbances, and disordered eating: investigating race, socioeconomic status, acculturation, and self-objectification as mediators. Diss Abstr Int. 2003;59(3-B):1360.
20 Hill MS. Examining objectification theory: sexual objectification's link with self-objectification and moderation by sexual orientation and age in White women. Diss Abstr Int. 2003;63(7-B):3515.

21 Heinberg LJ, Thompson JK, Stormer S. Development and validation of the Sociocultural Attitudes Towards Appearance Questionnaire. Int J Eat Disord. 1995 Jan;17(1):81-9.

22 Garner DM. Eating Disorder Inventory-2: professional manual. Psychological Assessment Resources; 1991.

23 Clausen L, Rosenvinge JH, Friborg O, Rokkedal K. Validating the eating disorder inventory-3 (EDI-3): A comparison between 561 female eating disorders patients and $878 \mathrm{fe}$ males from the general population. J Psychopathol Behav Assess. 2011 Mar;33(1):101-10.

24 Mussap AJ. Acculturation, body image, and eating behaviours in Muslim-Australian women. Health Place. 2009 Jun; 15(2):532-9.

25 Ferraro KF. Firm believers? Religion, body weight, and well-being. Rev Relig Res. 1998; 39(3):224-44.

26 Fredrickson BL, Roberts TA, Noll SM, Quinn DM, Twenge JM. That swimsuit becomes you: sex differences in self-objectification, restrained eating, and math performance. J Pers Soc Psychol. 1998 Jul;75(1):269-84.

27 Susov SL. The relationship between religious orientation, age, and eating disorder symptoms. BYU Scholars Archive. 2012; Paper 3157.

28 Nasser M. The new veiling phenomenon - is it an anorexic equivalent? A polemic. J Community Appl Soc Psychol. 1999;9(6):407-12.

29 Augustus-Horvath CL, Tylka TL. A test and extension of objectification theory as it predicts disordered eating: does women's age matter? J Couns Psychol. 2009;56(2):253-65. 